\title{
UPDATED RISK AREAS FOR TICK BORNE ENCEPHALITIS IN GERMANY
}

\author{
Editorial team (eurosurveillance@ecdc.europa.eu) ${ }^{1}$ \\ 1. European Centre for Disease Prevention and Control, Stockholm, Sweden
}

In its issue on 4 May 2009, the Epidemiologisches Bulletin of the Robert Koch-Institut (RKI), Germany, published an updated map of the risk areas for tick borne encephalitis (TBE) in Germany. TBE risk areas are areas in which TBE is endemic and where, in agreement with experts, a risk exists for people exposed to ticks that justify preventive measures. Such measures include vaccination for locals exposed to ticks, for tourists travelling to risk areas and people with occupational exposure.

The updated map shows that there is a risk of contracting the TBE virus through tick bites in the southern parts of Germany, in particular in the states Baden-Württemberg and Bavaria,

The TBE risk map is based on cases mandatory notification of TBE cases reported to the RKI in the period from $2002-2008$. In this period there were 1,917 TBE cases notified in total with the annual number ranging from 238 to 546 cases and 288 cases notified in 2008 . Five year reporting periods (2002 - 2006, 2003 - 2007, 2004 - 2008), were used to generate five year TBE incidences at the district level. To minimize the probability of underestimating the risk of infection in a particular district with increasing vaccination coverage, the number of reported cases in the so called district region, consisting of the district in question plus the adjacent districts, was created. A district was defined as a risk area if the incidence in the district or the district region was significantly ( $p$-value $<0.05$ ) higher than 1 per 100,000 inhabitants over a five year period.

In 2008, 136 districts were classified as TBE risk areas, four of them for the first time:

- 42 districts in Baden-Württemberg (1 additional district);

- 78 districts in Bavaria (3 additional districts);

- 8 districts in Hesse (unchanged);

- 7 districts in Thuringia (unchanged); and

- 1 district in Rhineland-Palatinate (unchanged).

The accompanying report summarises the TBE risk according to federal state as follows:

- federal states with defined TBE risk areas: Baden-Württemberg, Bavaria, Hesse, Rhineland-Palatinate, Thuringia;

- federal states with isolated cases of autochtonous TBE, in which no district fulfils the criteria of a TBE risk area: Brandenburg, Mecklenburg-West Pomerania, Lower Saxony North RhineWestpfalia, Saarland, Saxony, Saxony-Anhalt
- federal states in which no TBE cases have been diagnosed: Schleswig-Holstein, Hamburg, Bremen, Berlin.

Furthermore, it mentions that a slow but steady extension of the TBE risk areas in the past years is mainly limited to the southern federal states.

Surveillance and reporting of TBE cases are seen as the most efficient and cost effective basis on which to base preventive measures. However, should the incidence of cases drop due to the increase in vaccination, supplementary indicators for the risk of capturing TBE may become necessary in the future according to $\mathrm{RKI}$. In this context, data on vaccination coverage, establishment of systematic surveillance of the number of ticks, the ratio of ticks carrying the TBE virus or the number of animals infected, become important.

Based on the above mentioned epidemiological criteria, the German Standing Vaccination Committee (STIKO) recommends TBE vaccination for people who live in districts where the risk of exposure to ticks is established. Vaccination against TBE is recommended by the local health authorities in Baden-Württemberg, regardless of district (only two districts are not classified as risk areas).

\section{References}

1. Robert Koch-Institut. [TBE: Risk Areas in Germany] [In German] Epid Bull 2009;18:165-72

This article was published on 11 June 2009.

Citation style for this article: Editorial team. Updated Risk areas for tick borne encephalitis in Germany. Euro Surveill. 2009;14(23):pii=19236. Available online: http:/ www.eurosurveillance.org/ViewArticle.aspx?ArticleId=19236 